\title{
Amtolmetin: A Reappraisal of NSAID with Gastroprotection
}

\author{
Amit Garg, Ahsan Shoeb, Latha Subramanya Moodahadu, Akhilesh Sharma, \\ Arul Gandhi, and Shyam Akku
}

Global Medical Affairs, Dr. Reddy's Laboratories Ltd., Hyderabad 500034, India

Correspondence should be addressed to Shyam Akku; ashyamprasad@drreddys.com

Received 16 November 2015; Accepted 6 March 2016

Academic Editor: Bruce M. Rothschild

Copyright (C) 2016 Amit Garg et al. This is an open access article distributed under the Creative Commons Attribution License, which permits unrestricted use, distribution, and reproduction in any medium, provided the original work is properly cited.

\begin{abstract}
Aim. To assess the gastrosparing effect of amtolmetin guacyl (AMG) against other nonsteroidal anti-inflammatory drugs (NSAIDs) in patients with osteo-/rheumatoid arthritis. Methods. A literature search was done in the electronic databases (PubMed, Google Scholar, Embase, and Scopus) with key words "amtolmetin guacyl", "amtolmetin", and "arthritis"; filters were applied to obtain publications between 01-Jan-1985 and 01-Oct-2015, which were "clinical trials" in osteo-/rheumatoid arthritis patients and in "English language." Studies were assessed using the Jadad criteria and trials with score $\geq 3$ were included in the analysis to compare the safety and efficacy of AMG against other NSAIDs. Results. Search yielded 19 publications of which 3 were included for analysis. Baseline characteristics of patients were comparable between the AMG group and other NSAIDs (diclofenac, celecoxib, and piroxicam) groups in all trials. Efficacy of AMG was similar to the other NSAIDs compared in the trials. The number of adverse events (AEs) reported was similar between both the groups; however, severe AEs reported were significantly lower in the AMG group. Of note was the significant lower number of duodenal ulcers after treatment in the AMG group. Conclusions. AMG has efficacy similar to other NSAIDs and a safer gastrointestinal AE profile when compared to the other NSAIDs.
\end{abstract}

\section{Introduction}

Nonsteroidal anti-inflammatory drugs (NSAIDs) are extensively used in the treatment of chronic painful conditions such as rheumatoid arthritis (RA) and osteoarthritis (OA) to alleviate pain and inflammation associated with the disease and have become the mainstay of therapy in these conditions $[1,2]$. Major limiting factor in long-term use of NSAIDs is their safety, mainly gastrointestinal (GI) adverse effects ranging from mild to severe dyspeptic symptoms to the development of gastric or duodenal ulceration, hemorrhage, or perforation, which adversely affect patient's quality of life [1-3]. These complications indicate a clear unmet need in the safety of current treatment options for the management of RA and OA. NSAIDs which are more gastric tolerable are to be preferred for long-term use in these conditions. One such compound is amtolmetin guacyl (2-methoxyphenyl-1-methyl-5-p-methylbenzoyl-pyrrole2 -acetamido acetate, AMG) derived from the fusion between tolmetin with guaiacol and glycine, which is a nonselective NSAID having a cyclooxygenase-2/cyclooxygenase-1 (COX2/COX-1) selectivity ratio of 4.4 [4].
Amtolmetin guacyl (AMG) was demonstrated to be an effective anti-inflammatory drug with better GI tolerability profile displaying substantially lower incidence of GI adverse events compared to traditional NSAIDs $[5,6]$. This gastric sparing effect of AMG has been attributed to the presence of vanillic moiety in the molecule, which stimulates capsaicin receptors and releases calcitonin gene related peptide (CGRP) and consequently increases nitric oxide (NO) production [7], hence counterbalancing the deleterious effects of prostaglandin depletion due to COX inhibition and providing mucosal protection. Despite these favorable characteristics, its use has been limited owing to availability of newer NSAIDs. We analyzed earlier studies and compared the GI sparing effect of AMG with other widely used NSAIDs in patients with OA and RA to offer pragmatic suggestions for clinical practice.

\section{Methods}

A literature search was done in the electronic databases (PubMed, Google Scholar, Embase, and Scopus) with the following key words: "amtolmetin guacyl", "amtolmetin", 


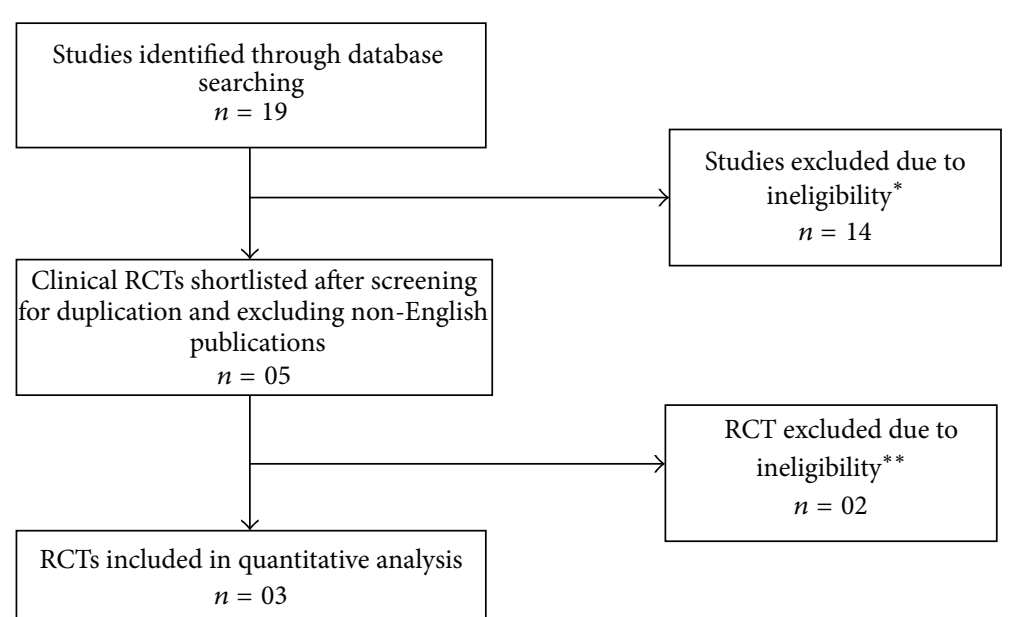

Figure 1: Literature search results. ${ }^{*}$ Preclinical studies, nonrandomized trials. ${ }^{* *}$ Jadad score 2, no significant gastrointestinal components discussed.

and "arthritis"; appropriate filters were applied in order to obtain publications between 01-Jan-1985 and 01-Oct-2015, which were "clinical trials," conducted in patients with osteo-/rheumatoid arthritis and in "English language."

Search result is shown in Figure 1. The quality of each randomized clinical trial was assessed using the Jadad criteria and trials with score $\geq 3$ were considered for the analysis [23]. Jadad score is a five-point score (1: low; 5: high quality) which was developed by Jadad AR in 1996 and is well validated for trials involving pain therapy. The Jadad criteria are simple and easy to use criteria that incorporate the most important individual components of methodological quality which includes randomization, blinding, and handling of patient attrition. However, it is limited by placing greater emphasis on the quality of reporting as opposed to the actual methodological quality of a trial. Olivo et al. [24] found that the Jadad scale demonstrated the strong evidence in terms of validity and reliability. Results from these shortlisted trials were computed to compare the safety of AMG against various other NSAIDs, with a focus on the GI adverse events.

2.1. Statistical Analysis. Data was captured in Microsoft Office Excel worksheet. Descriptive analysis was performed for all demographic variables. Student's $t$-test, Chi-square test, and post hoc test were used to test the hypothesis. The statistical tests were two-sided at the $5 \%$ level of significance. Statistical analysis was performed by using STATA version 13 for Windows (Stata Corp., College Station, TX).

\section{Results}

Initial search result yielded 19 publications of which only 4 met our selection criteria (Table 1). Of the 4 studies, 3 studies were considered for analysis and one study was excluded as gastrointestinal adverse effects were not discussed. The baseline characteristics of patients were comparable between the AMG and the other NSAIDs (Diclofenac, Celecoxib, and Piroxicam) groups in all these trials (Table 2). Efficacy of AMG was similar to the other NSAIDs compared in these trials. The numbers of adverse events (AEs) recorded was similar between both groups (Table 3); however, severe adverse events reported were significantly lower in the AMG group. Of note was the significant lower number of duodenal ulcers after treatment in the AMG group.

From these selected studies, the number of patients evaluated was 166 (48.3\%) in AMG group and 177 (51.6\%) in other NSAIDs' group. These patients were diagnosed to have either OA or RA. Females outnumbered men in both groups, 137 versus 29 (82.5\% versus 17.47\%) in AMG group and 141 versus 36 (79.7\% versus $20.3 \%$ ) in other NSAIDs' group. Mean age $( \pm \mathrm{SD})$ of patients was $58.97( \pm 3.2)$ in AMG group and $57.83( \pm 3.0)$ years in other NSAIDs' group.

Overall, gastrointestinal symptoms were seen in 80 (48.2\%) and 91 (51.4\%) patients in AMG and other NSAIDs' group, respectively. Table 3 shows GI symptoms reported from these three studies. There was no statistically significant difference in the occurrence of gastric symptoms in these two groups, but a significant lower mean percentage of severe gastric and/duodenal ulcer after treatment was observed with AMG. Withdrawal from the study due AEs was 5 (3\%) in AMG and 15 (8.5\%) in other NSAIDs' group, which was statistically significant $(p<0.05)$. There was a significant difference in mean percentage of serious AE leading to withdrawal from the study between these two groups favoring the AMG group. We also noted that there was a statistically significant difference in mean percentage of high endoscopy score between AMG (21.4\%) and other NSAIDs (27.6\%, $p<$ $0.05)$.

\section{Discussion}

NSAIDs are among the most commonly used drugs in the world. In Europe, NSAIDs represent more than $7.7 \%$ of all prescriptions and in 2004 a total of 111 million NSAID prescriptions were written in the United States alone $[1,2]$. Due to the noticeable efficacy and affordability, majority of patients with chronic painful conditions such as RA and 
TABLE 1: List of all studies considered and excluded with Jadad scores and reasons for exclusion.

\begin{tabular}{llcl}
\hline Serial number & Reference & Jadad score & Reason for exclusion \\
\hline 1 & Jajić et al. [4] & 3 & - \\
2 & Montrone et al. [6] & 3 & - \\
3 & Bianchi Porro et al. [8] & 3 & - \\
4 & Niccoli et al. [9] & 3 & No significant gastrointestinal components discussed \\
5 & Lazzaroni et al. [5] & 2 & Study conducted in healthy volunteers \\
6 & Kirkova et al. [10] & - & In vivo study \\
7 & Sostres et al. [11] & - & Not a RCT \\
8 & Pisano et al. [7] & - & Preclinical study \\
9 & Coruzzi et al. [12] & - & Preclinical study \\
10 & Tubaro et al. [13] & - & Preclinical study \\
11 & Tubaro et al. [14] & - & Preclinical study \\
12 & Patrignani et al. [15] & - & Not a RCT \\
13 & Li et al. [16] & - & Preclinical study \\
14 & Rong et al. [17] & - & Preclinical study \\
15 & Allison et al. [18] & - & Not a RCT \\
16 & Riezzo et al. [19] & - & Study in healthy volunteers; GI adverse effects not discussed \\
17 & Vicari et al. [20] & - & Not a RCT, published in Italian \\
18 & Morini et al. [21] & - & Preclinical study \\
19 & Hotha et al. [22] & - & Preclinical study \\
\hline
\end{tabular}

TABLE 2: Demographic profile of the patients from shortlisted studies.

\begin{tabular}{|c|c|c|c|c|c|c|c|c|}
\hline & \multicolumn{2}{|c|}{$\begin{array}{c}\text { Bianchi Porro et al. [8] } \\
\text { (4 weeks) }\end{array}$} & \multicolumn{2}{|c|}{$\begin{array}{c}\text { Montrone et al. [6] } \\
\text { (30 days) }\end{array}$} & \multicolumn{2}{|c|}{$\begin{array}{r}\text { Jajić et al. [4] } \\
\text { (24 weeks) }\end{array}$} & \multicolumn{2}{|c|}{ Age (year) mean $\pm S D$} \\
\hline & AMG & Diclofenac & AMG & Piroxicam & AMG & Celecoxib & AMG & NSAIDs \\
\hline Patients evaluated, $n$ & 32 & 32 & 49 & 50 & 85 & 95 & & \\
\hline Male, $n(\%)$ & $6(18.8)$ & $5(15.6)$ & $5(10.2)$ & $10(20)$ & $18(21.2)$ & $21(22.1)$ & $58.0 \pm 3.2$ & $57.8 \pm 3.0$ \\
\hline Female, $n(\%)$ & $26(81.2)$ & $27(84.4)$ & $44(89.8)$ & $40(80)$ & $67(78.8)$ & $74(77.9)$ & & \\
\hline
\end{tabular}

OA are managed by NSAIDs. Gastric intolerability is the most important and frequent adverse event and disadvantage of the current NSAIDs which may compel the patient to discontinue therapy [3]. With long-term usage of NSAIDs, development of GI related morbidities is apparent. Nearly $25 \%$ patients develop endoscopically visible ulcers after 3 months of NSAID usage and nearly $10 \%$ of patients in NSAID clinical trials withdraw from the trials because of adverse events $[25,26]$. Hence, in clinical practice, gastric acid neutralizers are coprescribed along with NSAIDs to manage GI adverse effects and for better patient compliance which is an additional financial burden for these patients. There is an unmet medical need for analgesics that have a better safety profile and can be used on a long-term basis for chronic pain management. Thus, it would be appropriate to identify an analgesic and anti-inflammatory compound with better gastric tolerability.

Amtolmetin has demonstrated efficacy similar to other NSAIDs with a better gastric sparing effect in various studies. Unlike other NSAIDs, AMG is preferentially administered on an empty stomach, as the maximum activation of gastric capsaicin receptors takes place on empty stomach. We identified studies that evaluated AMG and shortlisted these based on the Jadad score to compare the GI tolerability with other NSAIDs in patients with RA or OA.

Demographically, female patients were more in these studies analyzed. Our analysis of these selected studies supports the gastric sparing effect of AMG, which is further proved by endoscopic findings in two studies. Significantly high endoscopy score was seen in other NSAIDs' group (27.6\%) compared to AMG (21.4\%; $p<0.05)$. Though the number of AEs was similar among both the groups, the number of patients with severe gastric and/or duodenal ulcer after treatment was significantly more in other NSAIDs' group $(14.2 \%)$ compared to AMG $(4.3 \%$; $p<0.05)$.

Withdrawals due to GI adverse effects were more common in other NSAIDs' group than in AMG, indicating that the latter can contribute to better patient compliance. This in turn may result in improved symptom-free intervals, thus yielding better quality of life and productivity.

Literature and results of our analysis suggest that AMG's gastric sparing effect can overcome the limitation associated with long-term use of NSAID especially in painful chronic inflammatory disorders. With the increasing GI safety concerns with other NSAIDs, AMG seems to be a feasible treatment option. 
TABLE 3: Comparisons of adverse events in different trials.

\begin{tabular}{|c|c|c|c|c|c|c|c|}
\hline & \multicolumn{2}{|c|}{ Bianchi Porro et al. [8] } & \multicolumn{2}{|c|}{ Montrone et al. [6] } & \multicolumn{2}{|c|}{ Jajić et al. [4] } & \multirow{2}{*}{$p$ value } \\
\hline & AMG & Diclofenac & AMG & Piroxicam & AMG & Celecoxib & \\
\hline Patients evaluated, $n$ & 32 & 32 & 49 & 50 & 85 & 95 & \\
\hline GI symptoms, $n(\%)$ & $17(53.1)$ & $14(43.8)$ & $18(36.7)$ & $20(40)$ & $45(52.9)$ & $57(60)$ & $>0.05$ \\
\hline $\begin{array}{l}\text { Serious AEs related to the drug leading to } \\
\text { withdrawal, } n(\%)\end{array}$ & $3(9.4)$ & $5(15.6)$ & $2(4.1)$ & $9(18)$ & $0(0)$ & $1(1.1)$ & $<0.05^{1}$ \\
\hline $\begin{array}{l}\text { Cases of severe gastric and/or duodenal ulcer } \\
\text { after treatment, } n(\%)\end{array}$ & $1(3.1)$ & $8(25)$ & \multicolumn{2}{|c|}{$\mathrm{NE}$} & $4(4.7)$ & $10(10.5)$ & $<0.05$ \\
\hline Patients with very high endoscopy score, $n$ (\%) & $4(12.5)$ & $14(43.8)$ & \multicolumn{2}{|c|}{$\mathrm{NE}$} & $21(24.7)$ & $21(22.1)$ & $<0.05$ \\
\hline
\end{tabular}

NE: not evaluated.

${ }^{1}$ Additionally post hoc test was performed which gave similar results.

Though AMG has been approved for the treatment of painful disorders in Italy (2008), Russia (2014), and India (2008), there is paucity of data and it is underutilized in clinical practice. Long-term studies on large number of patients can provide further data on safety and its effect on quality of life in patients with painful chronic inflammatory conditions.

\section{Conclusions}

Amtolmetin guacyl with its established efficacy that is comparable to routinely prescribed NSAIDs is worth considering for chronic pain management in patients with osteoarthritis or rheumatoid arthritis. It has a good safety profile, particularly with its gastrointestinal sparing effect that can improve treatment compliance in these patients.

\section{Competing Interests}

All authors are employees of Dr. Reddy's Laboratories Ltd., Hyderabad, India.

\section{References}

[1] J. L. Goldstein, G. M. Eisen, N. Agrawal, W. F. Stenson, J. D. Kent, and K. M. Verburg, "Reduced incidence of upper gastrointestinal ulcer complications with the COX-2 selective inhibitor, valdecoxib," Alimentary Pharmacology and Therapeutics, vol. 20, no. 5, pp. 527-538, 2004.

[2] R. A. Moore, S. Derry, G. T. Makinson, and H. J. McQuay, "Tolerability and adverse events in clinical trials of celecoxib in osteoarthritis and rheumatoid arthritis: systematic review and meta-analysis of information from company clinical trial reports," Arthritis Research \& Therapy, vol. 7, no. 3, pp. R644R665, 2005.

[3] V. H. Chong and C. L. Wang, "Higher prevalence of gastrointestinal symptoms among patients with rheumatic disorders," Singapore Medical Journal, vol. 49, no. 5, pp. 419-424, 2008.

[4] Z. Jajić, M. Malaise, K. Nekam et al., "Gastrointestinal safety of amtolmetin guacyl in comparison with celecoxib in patients with rheumatoid arthritis," Clinical and Experimental Rheumatology, vol. 23, no. 6, pp. 809-818, 2005.
[5] M. Lazzaroni, A. Anderloni, and G. Bianchi Porro, "The effects on gastroduodenal mucosa of a new nonsteroidal antiinflammatory drug, amtolmetin-guacyl, versus piroxicam in healthy volunteers: a short-term, double-blind, endoscopically controlled study," European Journal of Gastroenterology and Hepatology, vol. 13, no. 7, pp. 833-839, 2001.

[6] F. Montrone, S. Santandrea, I. Caruso et al., "Amtolmetin guacyl versus piroxicam in patients with osteoarthritis," Journal of International Medical Research, vol. 28, no. 2, pp. 91-100, 2000.

[7] C. Pisano, D. Grandi, G. Morini et al., "Gastrosparing effect of new antiinflammatory drug amtolmetin guacyl in the rat: involvement of nitric oxide," Digestive Diseases and Sciences, vol. 44, no. 4, pp. 713-724, 1999.

[8] G. Bianchi Porro, F. Montrone, M. Lazzaroni, G. Manzionna, and I. Caruso, "Clinical and gastroscopic evaluation of amtolmetin guacyl versus diclofenac in patients with rheumatoid arthritis," Italian Journal of Gastroenterology and Hepatology, vol. 31, no. 5, pp. 378-385, 1999.

[9] L. Niccoli, S. Bellino, and F. Cantini, "Renal tolerability of three commonly employed non-steroidal anti-inflammatory drugs in elderly patients with osteoarthritis," Clinical and Experimental Rheumatology, vol. 20, no. 2, pp. 201-207, 2002.

[10] M. Kirkova, M. Kesiova, S. Konstantinova et al., "In vivo effects of amtolmetin guacyl on lipid peroxidation and antioxidant defence systems in different models of gastrointestinal injury," Autonomic and Autacoid Pharmacology, vol. 27, no. 1, pp. 63-70, 2007.

[11] C. Sostres, C. J. Gargallo, M. T. Arroyo, and A. Lanas, "Adverse effects of non-steroidal anti-inflammatory drugs (NSAIDs, aspirin and coxibs) on upper gastrointestinal tract," Best Practice and Research: Clinical Gastroenterology, vol. 24, no. 2, pp. 121132,2010

[12] G. Coruzzi, G. Coppelli, S. Spaggiari et al., "Gastroprotective effects of amtolmetin guacyl: a new non-steroidal antiinflammatory drug that activates inducible gastri nitric oxide synthase," Digestive and Liver Disease, vol. 34, no. 6, pp. 403410, 2002.

[13] E. Tubaro, L. Belogi, and C. M. Mezzadri, "The mechanism of action of amtolmetin guacyl, a new gastroprotective nonsteroidal anti-inflammatory drug," European Journal of Pharmacology, vol. 387, no. 2, pp. 233-244, 2000.

[14] E. Tubaro, L. Belogi, C. M. Mezzadri, and E. Bettelli, "Impact on the bowel of amtolmetin guacyl, a new gastroprotective non-steroidal anti-inflammatory drug," European Journal of Pharmacology, vol. 467, no. 1-3, pp. 173-183, 2003. 
[15] P. Patrignani, S. Tacconelli, A. Bruno, C. Sostres, and A. Lanas, "Managing the adverse effects of nonsteroidal antiinflammatory drugs," Expert Review of Clinical Pharmacology, vol. 4, no. 5, pp. 605-621, 2011.

[16] Y.-H. Li, J. Li, Y. Huang, X.-W. Lü, and Y. Jin, "Gastroprotective effect and mechanism of amtolmetin guacyl in mice," World Journal of Gastroenterology, vol. 10, no. 24, pp. 3616-3620, 2004.

[17] Z. Rong, Y. Xu, C. Zhang, D. Xiang, X. Li, and D. Liu, "Evaluation of intestinal absorption of amtolmetin guacyl in rats: breast cancer resistant protein as a primary barrier of oral bioavailability," Life Sciences, vol. 92, no. 3, pp. 245-251, 2013.

[18] M. C. Allison, A. G. Howatson, C. J. Torrance, F. D. Lee, and R. I. Russell, "Gastrointestinal damage associated with the use of nonsteroidal antiinflammatory drugs," The New England Journal of Medicine, vol. 327, no. 11, pp. 749-754, 1992.

[19] G. Riezzo, M. Chiloiro, and S. Montanaro, "Protective effect of amtolmetin guacyl versus placebo diclofenac and misoprostol in healthy volunteers evaluated as gastric electrical activity in alcohol-induced stomach damage," Digestive Diseases and Sciences, vol. 46, no. 8, pp. 1797-1804, 2001.

[20] E. Vicari, T. Cataldo, A. Arancio, and R. D’Agata, "Male urogenital amicrobial phlogosis: effects of the treatment with amtolmetin guacyl on some sperm parameters," Archivio Italiano di Urologia e Andrologia, vol. 71, no. 4, pp. 211-221, 1999.

[21] G. Morini, E. Guaita, M. Lazzaretti, D. Grandi, and G. Coruzzi, "Morphological features of rat gastric mucosa after acute and chronic treatment with amtolmetin guacyl: comparison with non-selective and COX-2-selective NSAIDs," Digestion, vol. 68, no. 2-3, pp. 124-132, 2003.

[22] K. K. Hotha, V. B. Dasari, A. N. Shaik et al., "Species difference in the in vitro and in vivo metabolism of amtolmetin guacil," Arzneimittel-Forschung, vol. 60, no. 11, pp. 667-674, 2010.

[23] A. R. Jadad, R. A. Moore, D. Carroll et al., "Assessing the quality of reports of randomized clinical trials: is blinding necessary?" Controlled Clinical Trials, vol. 17, no. 1, pp. 1-12, 1996.

[24] S. A. Olivo, L. G. Macedo, I. C. Gadotti, J. Fuentes, T. Stanton, and D. J. Magee, "Scales to assess the quality of randomized controlled trials: a systematic review," Physical Therapy, vol. 88, no. 2, pp. 156-175, 2008.

[25] A. J. Kivitz, S. Nayiager, T. Schimansky, A. Gimona, H. J. Thurston, and C. Hawkey, "Reduced incidence of gastroduodenal ulcers associated with lumiracoxib compared with ibuprofen in patients with rheumatoid arthritis," Alimentary Pharmacology and Therapeutics, vol. 19, no. 11, pp. 1189-1198, 2004.

[26] J. L. Goldstein, G. M. Eisen, T. A. Burke, B. M. Peña, J. Lefkowith, and G. S. Geis, "Dyspepsia tolerability from the patients' perspective: a comparison of celecoxib with diclofenac," Alimentary Pharmacology and Therapeutics, vol. 16, no. 4, pp. 819-827, 2002. 


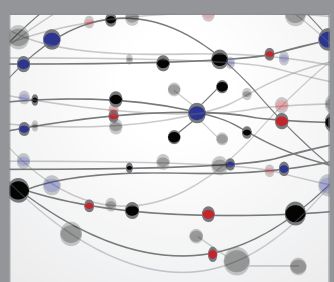

The Scientific World Journal
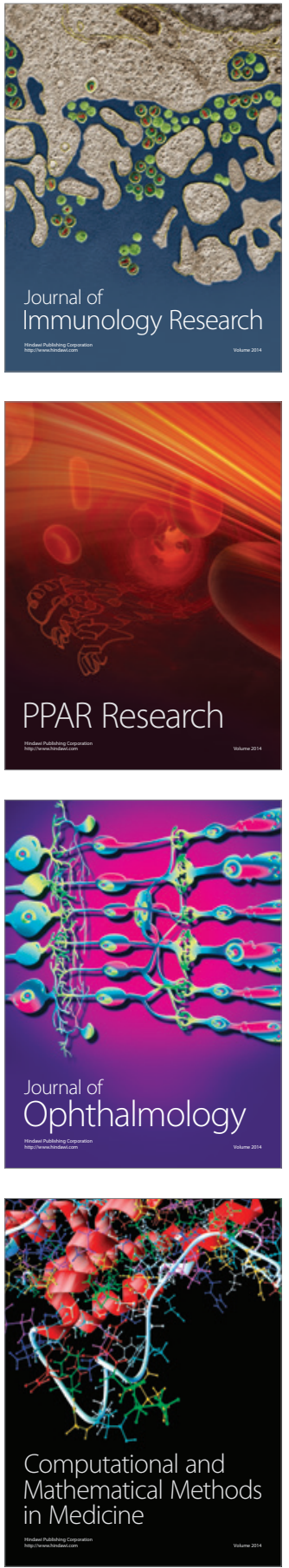

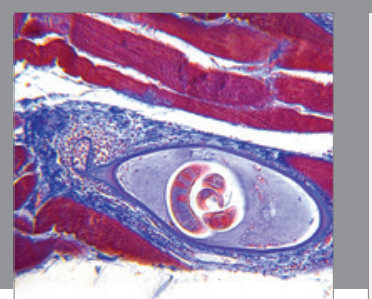

Gastroenterology Research and Practice

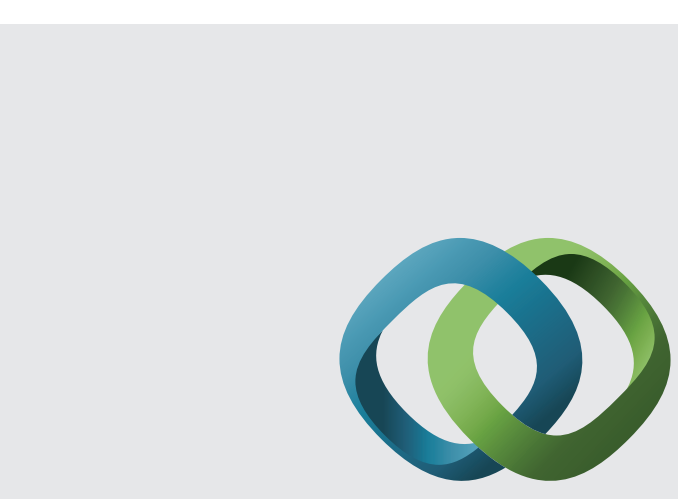

\section{Hindawi}

Submit your manuscripts at

http://www.hindawi.com
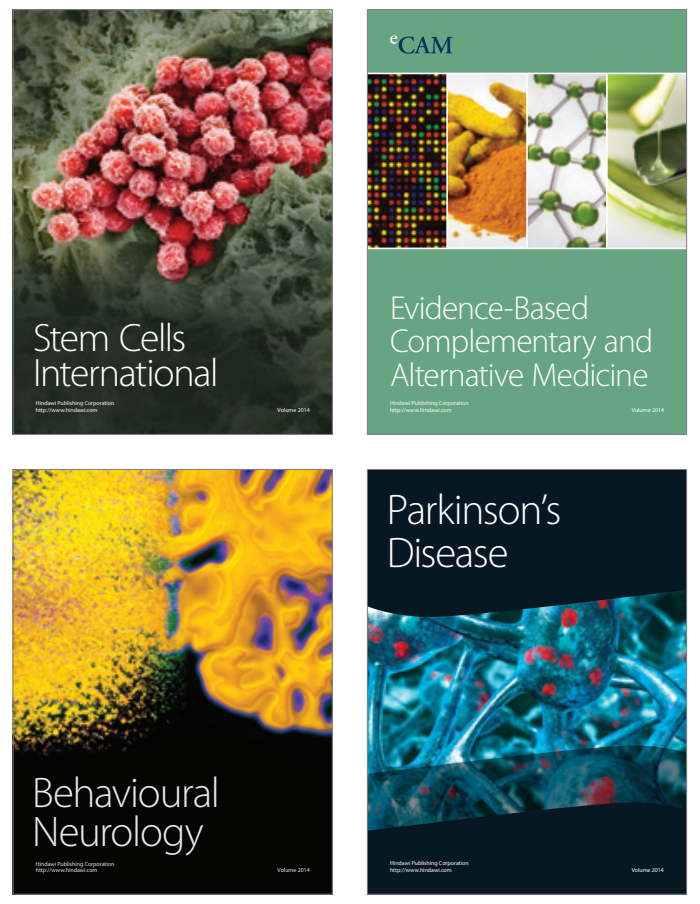
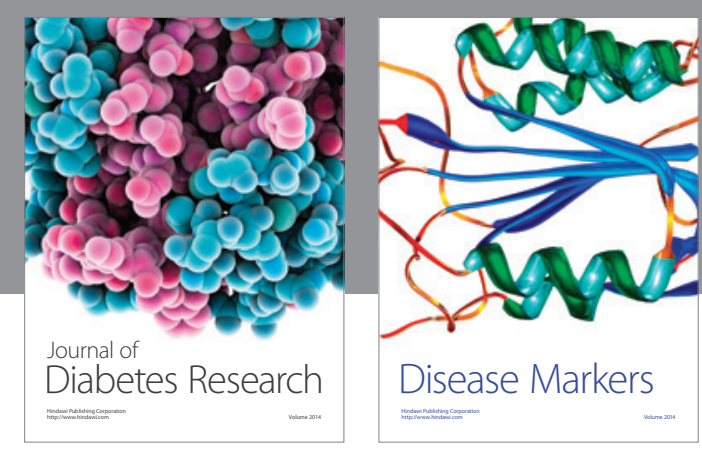

Disease Markers
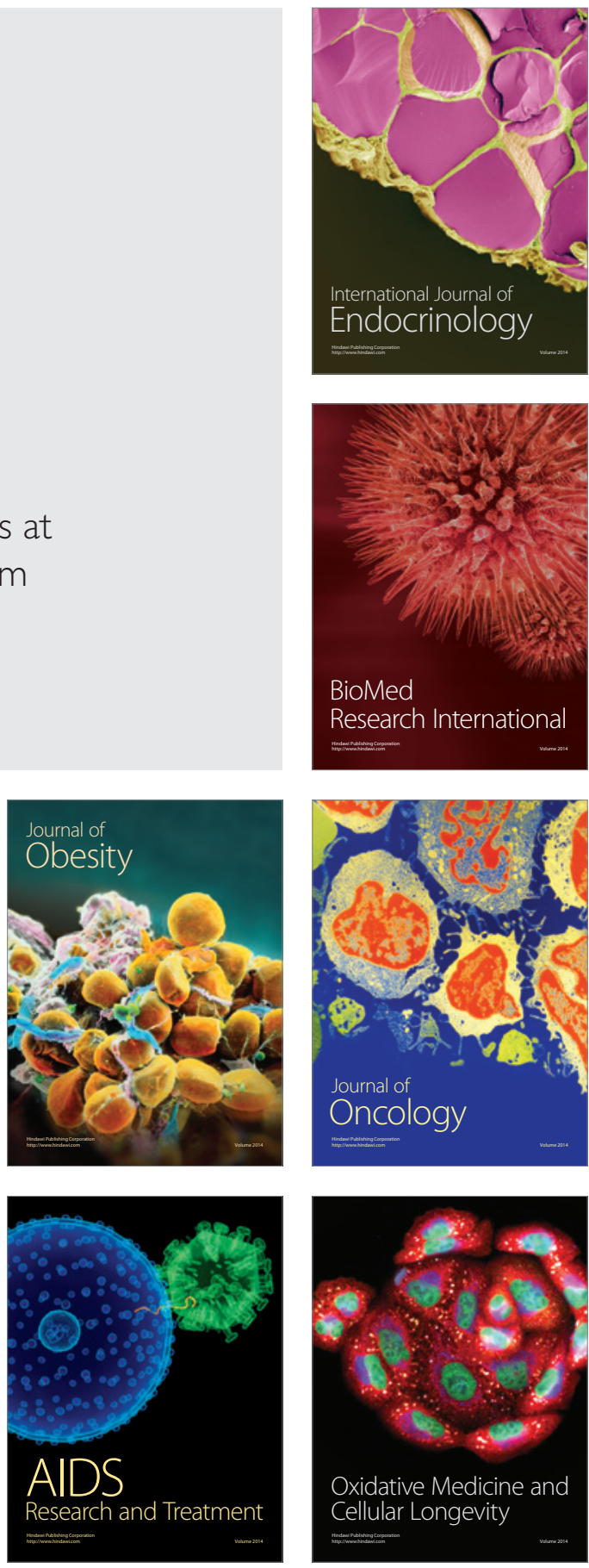